\title{
Vertebral body fracture rates after stereotactic body radiation therapy compared with external-beam radiation therapy for metastatic spine tumors
}

\author{
Enrique Vargas, BS, ${ }^{1,3}$ Matthew S. Susko, MD, MS, ${ }^{2}$ Praveen V. Mummaneni, MD, ${ }^{1}$ \\ Steve E. Braunstein, MD, PhD, ${ }^{2}$ and Dean Chou, MD' \\ Departments of ${ }^{1}$ Neurosurgery and ${ }^{2}$ Radiation Oncology and ${ }^{3}$ School of Medicine, University of California, San Francisco,
California
}

\begin{abstract}
OBJECTIVE Stereotactic body radiation therapy (SBRT) is utilized to deliver highly conformal, dose-escalated radiation to a target while sparing surrounding normal structures. Spinal SBRT can allow for durable local control and palliation of disease while minimizing the risk of damage to the spinal cord; however, spinal SBRT has been associated with an increased risk of vertebral body fractures. This study sought to compare the fracture rates between SBRT and conventionally fractionated external-beam radiation therapy (EBRT) in patients with metastatic spine tumors.

METHODS Records from patients treated at the University of California, San Francisco, with radiation therapy for metastatic spine tumors were retrospectively reviewed. Vertebral body fracture and local control rates were compared between SBRT and EBRT. Ninety-six and 213 patients were identified in the SBRT and EBRT groups, respectively. Multivariate analysis identified the need to control for primary tumor histology ( $p=0.003$ for prostate cancer, $p=0.0496$ for renal cell carcinoma). The patient-matched EBRT comparison group was created by matching SBRT cases using propensity scores for potential confounders, including the Spinal Instability Neoplastic Score (SINS), the number and location of spine levels treated, sex, age at treatment, duration of follow-up (in months) after treatment, and primary tumor histology. Covariate balance following group matching was confirmed using the Student t-test for unequal variance. Statistical analysis, including propensity score matching and multivariate analysis, was performed using $\mathrm{R}$ software and related packages.
\end{abstract}

RESULTS A total of 90 patients met inclusion criteria, with 45 SBRT and 45 EBRT matched cases. Balance of the covariates, SINS, age, follow-up time, and primary tumor histology after the matching process was confirmed between groups ( $p=0.062, p=0.174$, and 0.991, respectively, along with matched tumor histology). The SBRT group had a higher 5 -year rate of vertebral body fracture at $22.22 \%(n=10)$ compared with $6.67 \%(n=3)$ in the EBRT group $(p=0.044)$. Survival analysis was used to adjust for uneven follow-up time and showed a significant difference in fracture rates between the two groups $(p=0.044)$. SBRT also was associated with a higher rate of local control $(86.67 \%$ vs $77.78 \%)$.

CONCLUSIONS Patients with metastatic cancer undergoing SBRT had higher rates of vertebral body fractures compared with patients undergoing EBRT, and this difference held up after survival analysis. SBRT also had higher rates of initial local control than EBRT but this difference did not hold up after survival analysis, most likely because of a high percentage of radiosensitive tumors in the EBRT cohort.

https://thejns.org/doi/abs/10.3171/2020.5.SPINE191383

KEYWORDS stereotactic body radiation therapy; SBRT; external-beam radiation therapy; EBRT; fracture; spine tumor; patient matched; propensity score; Spinal Instability Neoplastic Score; SINS; oncology

$\mathrm{S}$ TEREOTACTIC body radiation therapy (SBRT) delivers highly conformal, dose-escalated radiation that conventionally fractionated external-beam radiation therapy (EBRT) does not. This affords a higher biologically effective dose (BED), a measure of the true biologi- cal dose delivered by a particular combination of dose per fraction and total dose, to the tumor with better sparing of the adjacent normal tissue. SBRT to metastatic spine lesions is associated with durable local control, with control rates of $80 \%-90 \%$ at 1 year. ${ }^{1-3}$ Additionally, SBRT is

ABBREVIATIONS BED = biologically effective dose; EBRT = external-beam radiation therapy; SBRT = stereotactic body radiation therapy; SINS = Spinal Instability Neoplastic Score; VCF = vertebral compression fracture.

SUBMITTED December 1, 2019. ACCEPTED May 11, 2020.

INCLUDE WHEN CITING Published online August 14, 2020; DOI: 10.3171/2020.5.SPINE191383. 
associated with good long-term pain control. ${ }^{4}$ Although there have been reports that SBRT is associated with posttreatment fractures, ${ }^{5-11}$ few studies have directly compared SBRT and EBRT. ${ }^{12,13}$ We report an analysis of our institutional experience with regard to rates of vertebral compression fractures (VCFs) and local control between SBRT and EBRT for metastases to the spine.

\section{Methods}

The committee on human research, our institutional review board, approved all research activities. Patients were identified through a search of adults undergoing radiotherapy between 2010 and 2017 at the University of California, San Francisco, with the ICD-10 code corresponding to secondary malignant neoplasm of bone (C79.51) and restricted to lesions of the spine. Medical records were reviewed retrospectively to include the Spinal Instability Neoplastic Score (SINS), patient age at treatment, sex, primary tumor histology, treatment location, and BED for each patient. SINS criteria include location (junctional, mobile spine, semirigid, or rigid), presence or absence of mechanical pain, type of bone lesion (lytic, mixed, or blastic), radiographic spine alignment, vertebral body collapse, and posterolateral involvement. Only patients with a minimum 5-month follow-up were included. Vertebral body fractures and disease progression were identified through treatment progress notes, outpatient clinic notes, and imaging studies, including radiographs and CT scans. Fractures were identified by radiology reports stating "new" or "worsening" fractures as defined by the radiologist. Vertebral body fracture and local control rates were compared between SBRT and EBRT groups. Progression of local disease was defined as a binary variable as any symptomatic disease progression resulting in an additional intervention or any symptomatic radiographic progression on imaging at the treated site. Patients with coexisting local disease progression, concurrent bone-modifying agent use, or mechanical deformities at the treatment site were excluded from this study. Although some patients had preexisting VCFs at other sites, only the areas of EBRT or SBRT were analyzed for new or worsening fractures; any VCFs distant from the radiated sites were not counted in this study. Any patients with prior methylmethacrylate augmentation at treatment levels were excluded from this study.

\section{Biologically Effective Dose}

BED was used as a measurement of the effect of dose delivery to patient tissue. It is a mathematical concept used to illustrate the biological effects from irradiation. In addition to being easy to compute, it is useful because 2 radiation treatments with the same BED produce the same radiobiological effects. ${ }^{14}$ BED was calculated according to the following standard formulation $(\mathrm{TD}=$ total dose in Gy, $\mathrm{d}=$ dose per fraction in Gy, and $\alpha / \beta=3$ and 10 for late and acute toxicities, respectively): $\mathrm{BED}=(\mathrm{TD})[1+\mathrm{d} /$ $(\alpha / \beta)]$. The number of treatment fractions and the dose per fraction were used to calculate the total dose (TD) in grays for each patient's BED calculation. The " $\alpha$ " represents the intrinsic radiosensitivity of the cells, defined as how many logs (to the exponential base "e") are effectively treated per gray. The " $\beta$ " represents the repairable portion of the radiation damage, requiring 6 hours or more for complete repair. The extent to which a tissue is radiosensitive or repairable (expressed in $\alpha / \beta$ ratio) is dependent on the primary tumor histology. These values can be identified from previously published $\alpha / \beta$ ratios for varying tumor types..$^{15}$ Although patients may not have the same radiation schedules, the total effect of different radiation schedules can be recalculated and expressed as a BED: $\mathrm{BED}_{10}$ for tumor and $\mathrm{BED}_{3}$ for normal tissues. ${ }^{15,16}$ Both $\mathrm{BED}_{10}$ and $\mathrm{BED}_{3}$ values (BED calculation with an $\alpha / \beta$ ratio of 3 and 10 , respectively) were calculated to account for early and late responding tissues.

\section{Propensity Matching}

Because more patients were treated with EBRT than SBRT overall, a propensity matching model was utilized to effectively compare the groups. Various studies have used propensity matching to reduce the baseline differences in patient characteristics between two groups and compare outcomes via univariate or multivariate analysis. ${ }^{17-19}$ The patient-matched EBRT comparison group was constructed by matching SBRT cases based on propensity scores for the following potentially confounding factors: SINS, spine levels treated, sex, age at treatment, duration of follow-up after treatment, and tumor histology. Exact matching was used for categorical variables and continuous variables when possible. Caliper matching and nearest-neighbor matching were used otherwise. Covariate balance following group matching was confirmed without significant difference between groups (Table 1). For statistical analysis, means of samples were compared using the Student t-test for unequal variance, with significance set at $p<0.05$. Propensity score matching was carried out using R statistical software and the associated "MatchIt" package (www.rproject.org). Survival analysis was also conducted on $\mathrm{R}$ using the Kaplan-Meier method, as part of the survival package, to account for uneven follow-up times.

\section{Results}

\section{Patient Demographics}

Of the total 309 identified patients who underwent SBRT and EBRT, 251 were available for propensity score matching. Only patients with at least 5 months of follow-up were considered for analysis. Propensity score matching resulted in 45 SBRT cases with 45 EBRT comparisons. Table 1 provides a summary of the clinical and treatment characteristics of the SBRT and EBRT groups. The mean age at the time of treatment was $59.97 \pm 11.69$ years (range 33-79 years) for the SBRT group and 56.67 \pm 11.20 years (range 29-82 years) for the EBRT group ( $\mathrm{p}=$ 0.174 ). Both groups had similar mean follow-up durations: $27.64 \pm 16.84$ months (range 5-63 months) for the SBRT group and $27.60 \pm 17.96$ months (range 6-82 months) for the EBRT group $(\mathrm{p}=0.991)$. The thoracic spine was the most common treatment location for both the SBRT and EBRT groups at $50.7 \%$ and $50 \%$ of cases, respectively (p $=0.931$ ). Both groups had similar rates of treatment to the lumbar spine: $36.62 \%$ and $37.18 \%$, respectively $(\mathrm{p}=0.913)$. 
TABLE 1. Summary of patient demographics and clinical characteristics

\begin{tabular}{|c|c|c|c|}
\hline Variable & SBRT & EBRT & $p$ Value \\
\hline Mean age at treatment, yrs & $59.97 \pm 11.69$ & $56.67 \pm 11.20$ & 0.174 \\
\hline Mean follow-up, mos & $27.64 \pm 16.84$ & $27.60 \pm 17.96$ & 0.991 \\
\hline Mean SINS & $8.68 \pm 1.96$ & $9.35 \pm 1.44$ & 0.062 \\
\hline \multicolumn{4}{|l|}{ Treatment location } \\
\hline Cervical & $9(12.68)$ & $10(12.82)$ & 0.957 \\
\hline Thoracic & $36(50.70)$ & $39(50.00)$ & 0.931 \\
\hline Lumbar & $26(36.62)$ & $29(37.18)$ & 0.913 \\
\hline Total level count* & 71 & 78 & \\
\hline Multilevel cases & $11(24.44)$ & $12(26.67)$ & 0.960 \\
\hline \multicolumn{4}{|l|}{ Sex } \\
\hline Male & $24(53.33)$ & $22(48.89)$ & 0.509 \\
\hline Female & $21(46.67)$ & $23(51.11)$ & 0.502 \\
\hline Mean $\mathrm{BED}_{10}$ & $64.75 \pm 46.55$ & $45.33 \pm 19.10$ & 0.063 \\
\hline Mean $\mathrm{BED}_{3}$ & $144 \pm 91.81$ & $80 \pm 48.11$ & 0.001 \\
\hline \multicolumn{4}{|l|}{ Primary tumor type } \\
\hline Breast & $15(33.33)$ & $15(33.33)$ & 1 \\
\hline NSCLC & $6(13.33)$ & $6(13.33)$ & 1 \\
\hline Prostate & $6(13.33)$ & $6(13.33)$ & 1 \\
\hline RCC & $7(15.56)$ & $7(15.56)$ & 1 \\
\hline $\mathrm{HCC}$ & $3(6.67)$ & $3(6.67)$ & 1 \\
\hline Pancreatic & $1(2.22)$ & $1(2.22)$ & 1 \\
\hline Thyroid & $3(6.67)$ & $3(6.67)$ & 1 \\
\hline Melanoma & $3(6.67)$ & $3(6.67)$ & 1 \\
\hline Other & $1(2.22)$ & $1(2.22)$ & 1 \\
\hline
\end{tabular}

$\mathrm{HCC}=$ hepatocellular carcinoma; NSCLC $=$ non-small cell lung cancer; $\mathrm{RCC}=$ renal cell carcinoma.

Data are presented as the number of patients (\%) unless stated otherwise. Mean values are presented as the mean \pm SD. Boldface type indicates statistical significance.

* Total count exceeds 45 because some patients were treated in multiple locations. This was taken into account when finding a suitable match.

There was a male predominance in the SBRT group, with $53.3 \%$ of patients self-identifying as male. There was a female predominance in the EBRT group, with $51.1 \%$ selfidentifying as female. Within each group, roughly $26 \%$ of patients underwent multilevel treatment. This was accounted for when creating matched patient pairs. Tables 2 and 3 provide coefficient results and patient characteristics for those having VCF, respectively.

\section{Local Disease Control and Vertebral Body Fracture Rates}

In the SBRT group, 39 patients $(86.67 \%)$ did not develop symptomatic disease progression within 5 years of treatment that resulted in an additional intervention or radiographic progression on imaging at the treated site compared with 35 patients $(77.78 \%)$ in the EBRT group. However, survival analysis showed no difference in local control rates between the SBRT group and the EBRT group (likelihood ratio $=0.7, p=0.404$ ). The SBRT group had a higher 5-year rate of vertebral body fractures at $22.22 \%$ $(n=10)$ compared with $6.67 \%(n=3)$ in the EBRT group.
TABLE 2. Summary of statistical results from multivariate analysis with fracture status and progression of disease as dependent variables

\begin{tabular}{lcc}
\hline \multicolumn{1}{c}{ Variable } & Coefficient & p Value \\
\hline Follow-up (mos) & 0.00152 & 0.599 \\
\hline BED $_{10}$ & 0.00197 & 0.601 \\
\hline BED $_{3}$ & 0.00065 & 0.670 \\
\hline SINS & 0.01291 & 0.561 \\
\hline Primary prostate cancer & 0.34577 & $\mathbf{0 . 0 0 3}$ \\
\hline Primary RCC & 0.22424 & $\mathbf{0 . 0 4 9}$ \\
\hline
\end{tabular}

Boldface type indicates statistical significance.

There was a significant difference in VCF rates between the SBRT and EBRT groups following survival analysis (likelihood ratio $=4.04, \mathrm{p}=0.044$ ). Kaplan-Meier survival plots comparing survival probability estimates for fractures and local control, respectively, are shown in Figs. 1 and 2 .

\section{Risk Factors}

Multivariate analysis identified the need to control for primary tumor histology at the start of treatment. Tumor location was determined on the basis of MRI, CT, or radiography findings. Radiation oncology notes from initial evaluations for radiation treatment were also utilized. Primary tumor histology was determined using past medical records and biopsy results. The F-test confirmed that the multivariate statistical model was significant $(p=0.0139)$, with a multiple $\mathrm{R}$ and adjusted $\mathrm{R}^{2}$ of 0.265 and 0.151 , respectively. Prostate cancer and renal cell carcinoma were both found to be significantly correlated with VCF, with coefficients of $0.346(p=0.003)$ and $0.224(p=0.049)$, respectively. A summary of results and coefficients from the multivariate analysis is provided in Table 2 . Factors included in the statistical model were follow-up time, SINS, tumor histology, age at treatment, and $\mathrm{BED}_{3} / \mathrm{BED}_{10}$. Exact matching was used for categorical variables and for

TABLE 3. Summary of patient and clinical characteristics for those experiencing a fracture

\begin{tabular}{lcc}
\hline \multicolumn{1}{c}{ Variable } & SBRT $(n=10)$ & EBRT $(n=3)$ \\
\hline Treatment of fracture & & 2 \\
\hline Surgery & 4 & 1 \\
\hline Palliative radiation & 5 & 0 \\
\hline Pain management & 1 & \\
\hline Type of tumor & & 0 \\
\hline Breast & 1 & 0 \\
\hline Thyroid & 1 & 3 \\
\hline Prostate & 1 & 0 \\
\hline RCC & 4 & 0 \\
\hline HCC & 2 & 0 \\
\hline Melanoma & 1 & 0 \\
\hline Other & 0 &
\end{tabular}




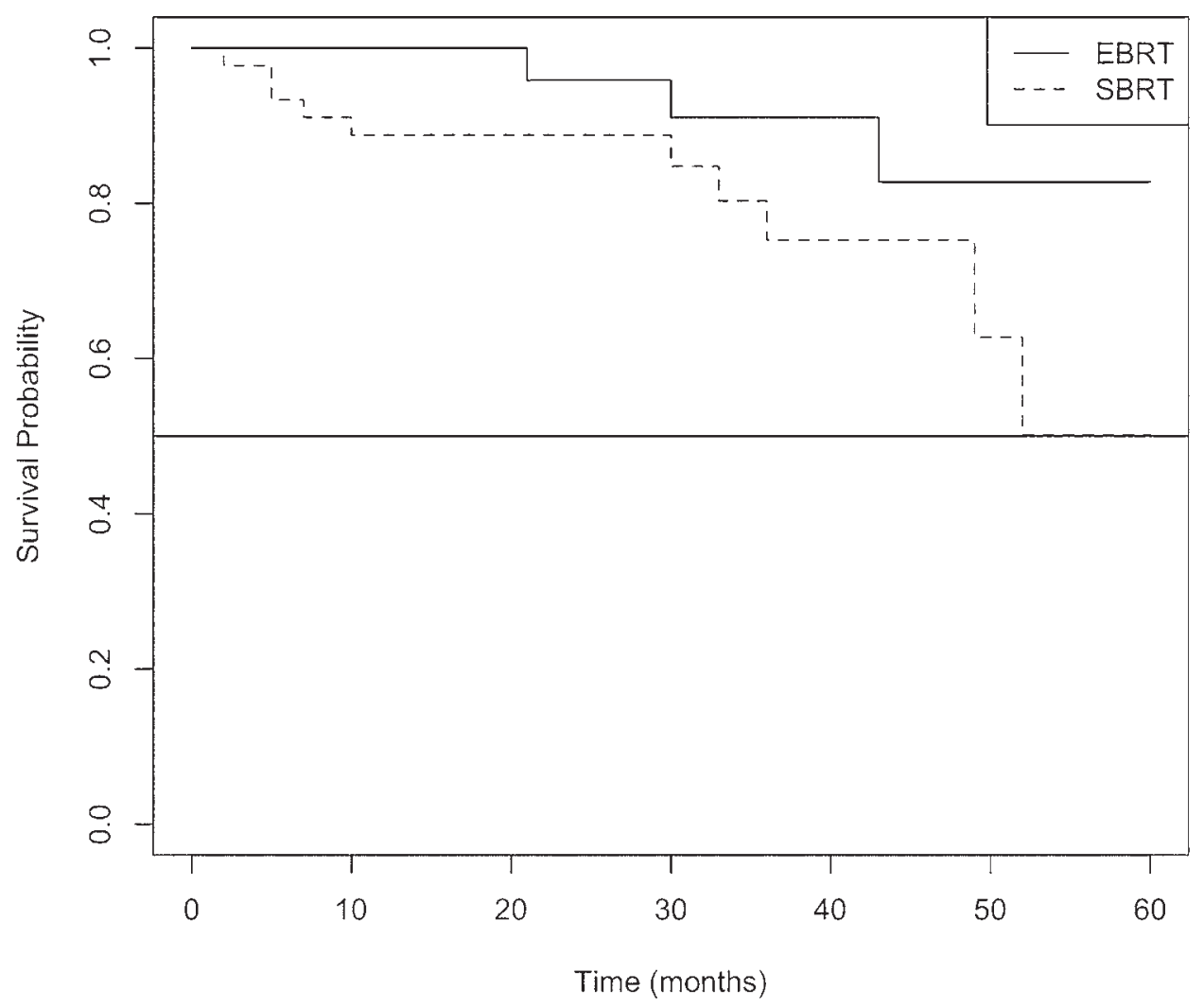

FIG. 1. Kaplan-Meier method comparing estimations of the survival probability of developing a VCF event for SBRT versus EBRT.

continuous variables when possible. Caliper matching and nearest neighbor matching were used otherwise. Controlling for the potentially confounding variables primary tumor histology and SINS did not affect outcome differences between groups. Variables analyzed included SINS, spine levels treated, sex, age at treatment, follow-up duration after treatment, and tumor histology.

\section{Discussion}

Treatment of metastatic spinal disease can widely vary from surgical treatment to chemotherapy, EBRT, or SBRT. The goals of treatment are to decrease clinical symptoms and afford local control of the tumor. ${ }^{10,20}$ Two factors that may complicate treatment and result in more pain are vertebral body fractures and progression of local disease. One disadvantage of SBRT is the risk of VCF; the SBRT group had a higher 5-year rate of fracture than the EBRT group at $22.22 \%$ versus $6.67 \%$. This difference was significant following survival analysis $(\mathrm{p}=0.044)$.

Over the past decade, numerous studies have confirmed the safety and efficacy of SBRT. SBRT is considered an effective treatment, and there is evidence that it provides improved local control compared with traditional EBRT in the treatment of metastatic spine disease and related pain. ${ }^{1,2,5,21-26}$ The present study observed local control rates similar to those in previously reported studies..$^{13}$ Although the local control rate in the SBRT cohort (86.67\%) was initially higher than that of the EBRT cohort (77.78\%), both control rates were similar following survival analysis. There are many possible reasons for this finding. It is possible that the large proportion of radiosensitive tumors (breast and prostate cancers made up $49 \%$ of cases) included in both cohorts makes the local control afforded by EBRT appear comparably effective because there are fewer radioresistant tumors. Similarly, it is plausible that fracture rates would have increased if the proportion of radioresistant tumors were higher, as a higher BED may have been required for treatment. ${ }^{9}$

However, there are also known adverse events associated with spinal SBRT, such as vertebral body fractures. ${ }^{9}$ To date, limited studies have directly compared outcomes of patients receiving SBRT versus conventional EBRT. Chang et al. evaluated clinical outcomes after SBRT for spinal metastasis compared with conventional radiation therapy, but they only evaluated local control in patients with hepatocellular carcinoma. ${ }^{12}$ Sohn et al. also compared outcomes between the two treatment modalities in a matched-pair study but only looked at a subset of 13 patients with renal cell carcinoma and did not compare fracture rates between groups. ${ }^{27}$ Previous studies have also reported risk factors for vertebral body fracture or disease progression in patients receiving SBRT, including the presence of a preexisting VCF, pain as the reason for treatment, smoking history, high doses per fraction (20 Gy or greater in a single fraction), or presence of kyphotic or scoliotic deformities. ${ }^{5,21,28-31}$

The goal of our study was to compare rates of vertebral 


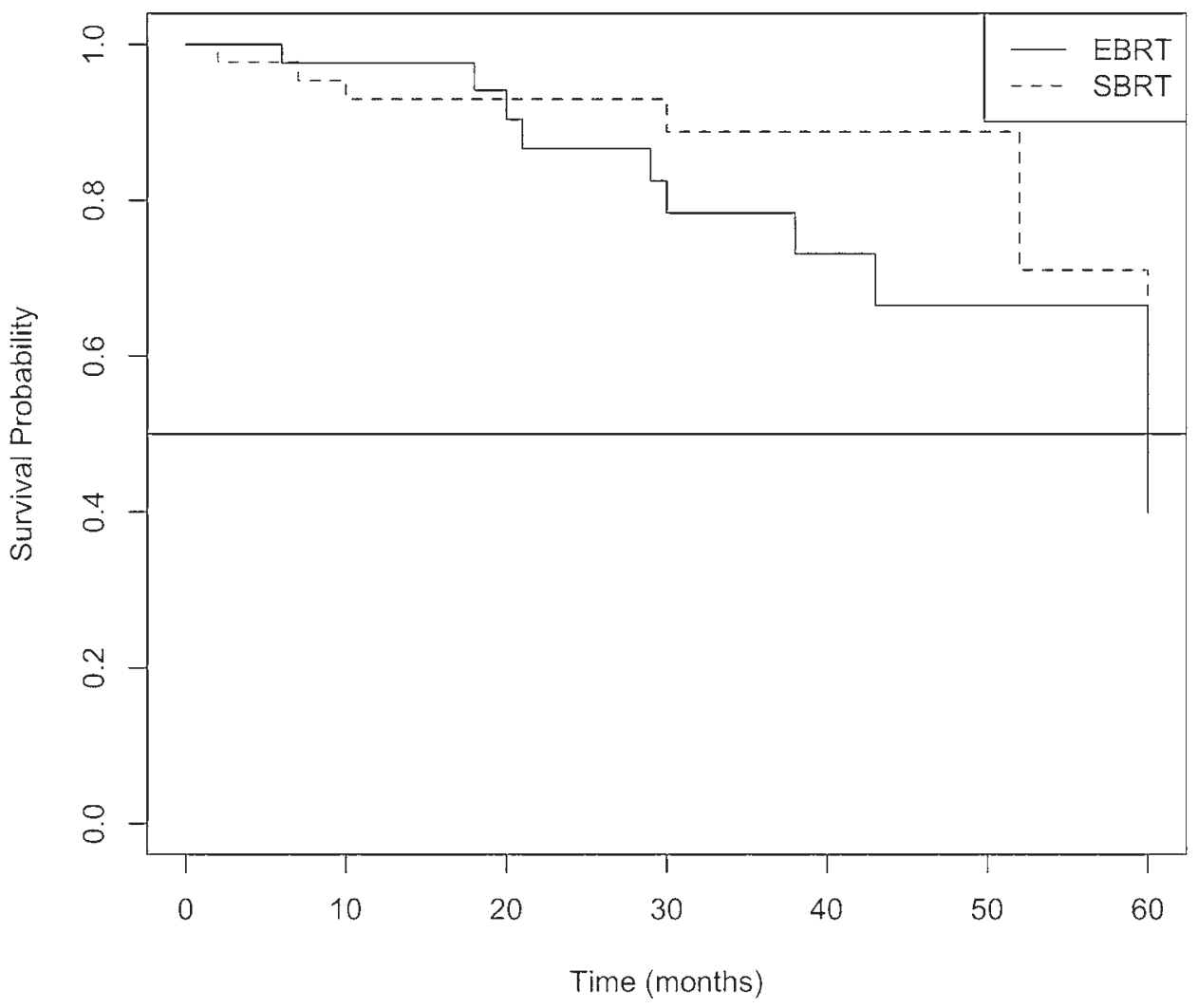

FIG. 2. Kaplan-Meier method comparing estimations of the survival probability of progression of disease for SBRT versus EBRT.

body fracture and local control as measured by progression of disease in patients treated with SBRT compared with EBRT for metastatic spine disease. Several studies have looked specifically at fracture rates and local control in patients receiving SBRT treatment, but there have been varying results across studies. For instance, Jawad et al. found the incidence of vertebral body fracture to be as low as $5.7 \%,{ }^{8}$ while other studies have cited fracture rates as high as $39 \% .{ }^{31}$ Most recent studies, however, have reported fracture rates around $10 \%-15 \% .5,7,21$ Among the 90 patients included in our study, regardless of treatment type, there were a total of 13 vertebral body fractures $(14.44 \%)$ and 16 total cases of local disease progression $(17.78 \%)$, which is consistent with the results of previous studies..$^{13}$ It is important to note, however, that the propensity matched nature of this analysis may not make our total fracture and local control rates representative of the true rates of either SBRT or EBRT. Propensity matching may select against types of tumor histology that are only or best treated with SBRT, thus eliminating these patients from analysis. This should be considered when interpreting the data. In addition, while a 1-year follow-up would be ideal, it would have dramatically decreased our statistical power given that the survival of metastatic disease can be less than a year.

We attempted to control for confounding variables between patients who underwent SBRT and patients treated via EBRT by using propensity score matching based on SINS, age at treatment, sex, tumor histology, treatment lo- cation, follow-up time, and BED. There have been reports recommending SBRT to be strongly considered as a treatment option in patients who require reirradiation treatment. ${ }^{32}$ Although there are data suggesting retreatment with SBRT can be effective, ${ }^{30}$ only patients with primary, initial treatment with either SBRT or conventional EBRT were included in our study to reduce effects of confounders. Covariate balance was confirmed without significant difference between groups following the matching process. Similar to previous studies, multivariate analysis confirmed that tumor histology was a significant predictor of fracture; thus, it was critical to match the two cohorts..$^{28}$ Survival analysis using the Kaplan-Meier method was also performed to account for the uneven follow-up time across groups. Despite these measures, it is important to acknowledge that there is no way to make these groups truly comparable, as EBRT and SBRT are generally used to treat two fundamentally different populations of patients. Building larger data sets and conducting multiinstitutional analysis could be pursued to minimize this bias.

There are various other limitations to this study. First, it is limited by its retrospective, single-institutional nature. Although the mean follow-up times were 28 and 27 months for the SBRT and EBRT groups, respectively, there were several patients with shorter follow-up times. Most fractures subsequent to SBRT typically develop in the first 1.5-3.5 months following treatment. ${ }^{10}$ However, in our experience, the average follow-up time at which a fracture occurred was 24 months after treatment began. 
Therefore, our rates of new or worsening vertebral fracture and progression of disease may be overestimated because of our longer follow-up time. In addition, the lack of objective assessment of pain with health-related quality-of-life measures hinders our ability to assess the true impact of these fractures beyond imaging and clinic notes.

Despite efforts to reduce effects of potential confounders via propensity score matching, there are still many variables not taken into account. For example, initial data collection yielded many more patients receiving EBRT ( $\mathrm{n}$ $=213)$ than SBRT $(n=96)$. A significant proportion of the SBRT cases could not be successfully matched to the EBRT cohort due to rare primary tumor histology, leading to only 45 successfully matched pairs. It is possible that these excluded cases may have changed our results. Another significant confounding variable is the selection bias for SBRT versus EBRT. It is difficult to account for the fact that SBRT or EBRT may be preferred for certain disease states regardless of any potential increased risk of fracture. There are many other factors that play into the decision of choosing one modality over another for any given patient. Some common factors include insurance restrictions covering EBRT but not SBRT, geographic location restricting patient choice (some patients could not travel to our medical center for SBRT treatment), radiation oncologist preferences, tumor histology, overall condition of the patient, and oncologist preferences. Nonetheless, there are limited reports directly comparing EBRT and SBRT and fracture rates, and, despite the aforementioned limitations, our findings are consistent with those of previous reports of potentially higher fracture rates with SBRT.

\section{Conclusions}

SBRT appears to be associated with a higher fracture rate compared with EBRT. The difference in fracture rates between the two groups was also significant following survival analysis. It is possible that the high proportion of radiosensitive tumors found in both cohorts may be responsible for similar local control rates between SBRT and EBRT. Even with propensity matching, the two groups may never be completely comparable because of exclusion of radioresistant tumors in the EBRT cohort.

\section{References}

1. Ahmed KA, Stauder MC, Miller RC, et al. Stereotactic body radiation therapy in spinal metastases. Int J Radiat Oncol Biol Phys. 2012;82(5):e803-e809.

2. Chang EL, Shiu AS, Mendel E, et al. Phase I/II study of stereotactic body radiotherapy for spinal metastasis and its pattern of failure. J Neurosurg Spine. 2007;7(2):151-160.

3. Wang XS, Rhines LD, Shiu AS, et al. Stereotactic body radiation therapy for management of spinal metastases in patients without spinal cord compression: a phase 1-2 trial. Lancet Oncol. 2012;13(4):395-402.

4. Puvanesarajah V, Lo SL, Aygun N, et al. Prognostic factors associated with pain palliation after spine stereotactic body radiation therapy. J Neurosurg Spine. 2015;23(5):620-629.

5. Balagamwala EH, Jung DL, Angelov L, et al. Incidence and risk factors for vertebral compression fractures from spine stereotactic body radiation therapy: results of a large institutional series. Int J Radiat Oncol. 2013;87(2):S89.

6. Boehling NS, Grosshans DR, Allen PK, et al. Vertebral com- pression fracture risk after stereotactic body radiotherapy for spinal metastases. J Neurosurg Spine. 2012;16(4):379-386.

7. Faruqi S, Tseng CL, Whyne C, et al. Vertebral compression fracture after spine stereotactic body radiation therapy: a review of the pathophysiology and risk factors. Neurosurgery. 2018;83(3):314-322.

8. Jawad MS, Fahim DK, Gerszten PC, et al. Vertebral compression fractures after stereotactic body radiation therapy: a large, multi-institutional, multinational evaluation. J Neurosurg Spine. 2016;24(6):928-936.

9. Sahgal A, Atenafu EG, Chao S, et al. Vertebral compression fracture after spine stereotactic body radiotherapy: a multiinstitutional analysis with a focus on radiation dose and the spinal instability neoplastic score. J Clin Oncol. 2013;31(27): 3426-3431.

10. Sung SH, Chang UK. Evaluation of risk factors for vertebral compression fracture after stereotactic radiosurgery in spinal tumor patients. Korean J Spine. 2014;11(3):103-108.

11. Virk MS, Han JE, Reiner AS, et al. Frequency of symptomatic vertebral body compression fractures requiring intervention following single-fraction stereotactic radiosurgery for spinal metastases. Neurosurg Focus. 2017;42(1):E8.

12. Chang UK, Kim MS, Han CJ, Lee DH. Clinical result of stereotactic radiosurgery for spinal metastasis from hepatocellular carcinoma: comparison with conventional radiation therapy. J Neurooncol. 2014;119(1):141-148.

13. Sprave T, Verma V, Förster R, et al. Local response and pathologic fractures following stereotactic body radiotherapy versus three-dimensional conformal radiotherapy for spinal metastases - a randomized controlled trial. BMC Cancer. 2018;18(1):859.

14. Voyant C, Julian D, Roustit R, et al. Biological effects and equivalent doses in radiotherapy: a software solution. Rep Pract Oncol Radiother. 2013;19(1):47-55.

15. van Leeuwen CM, Oei AL, Crezee J, et al. The alfa and beta of tumours: a review of parameters of the linear-quadratic model, derived from clinical radiotherapy studies. Radiat Oncol. 2018;13(1):96.

16. Fowler JF, Tomé WA, Fenwick JD, Mehta MP. A challenge to traditional radiation oncology. Int J Radiat Oncol Biol Phys. 2004;60(4):1241-1256.

17. Kim BD, Edelstein AI, Hsu WK, et al. Spine surgeon specialty is not a risk factor for 30-day complication rates in single-level lumbar fusion: a propensity score-matched study of 2528 patients. Spine (Phila Pa 1976). 2014;39(15):E919E927.

18. Mangona VS, Aneese AM, Marina O, et al. Toxicity after central versus peripheral lung stereotactic body radiation therapy: a propensity score matched-pair analysis. Int $\mathrm{J} \mathrm{Ra-}$ diat Oncol Biol Phys. 2015;91(1):124-132.

19. Smith JS, Lafage V, Shaffrey CI, et al. Outcomes of operative and nonoperative treatment for adult spinal deformity: a prospective, multicenter, propensity-matched cohort assessment with minimum 2-year follow-up. Neurosurgery. 2016;78(6): 851-861.

20. Haley ML, Gerszten PC, Heron DE, et al. Efficacy and cost-effectiveness analysis of external beam and stereotactic body radiation therapy in the treatment of spine metastases: a matched-pair analysis. J Neurosurg Spine. 2011;14(4): 537-542.

21. Balagamwala EH, Naik M, Reddy CA, et al. Pain flare after stereotactic radiosurgery for spine metastases. J Radiosurg SBRT. 2018;5(2):99-105.

22. Hall WA, Stapleford LJ, Hadjipanayis CG, et al. Stereotactic body radiosurgery for spinal metastatic disease: an evidencebased review. Int J Surg Oncol. 2011;2011:979214.

23. Husain ZA, Sahgal A, De Salles A, et al. Stereotactic body radiotherapy for de novo spinal metastases: systematic review. J Neurosurg Spine. 2017;27(3):295-302. 
24. Ozdemir Y, Torun N, Guler OC, et al. Local control and vertebral compression fractures following stereotactic body radiotherapy for spine metastases. J Bone Oncol. 2019;15: 100218.

25. Tseng CL, Eppinga W, Charest-Morin R, et al. Spine stereotactic body radiotherapy: indications, outcomes, and points of caution. Global Spine J. 2017;7(2):179-197.

26. Zeng KL, Tseng CL, Soliman H, et al. Stereotactic body radiotherapy (SBRT) for oligometastatic spine metastases: an overview. Front Oncol. 2019;9:337.

27. Sohn S, Chung CK, Sohn MJ, et al. Stereotactic radiosurgery compared with external radiation therapy as a primary treatment in spine metastasis from renal cell carcinoma: a multicenter, matched-pair study. J Neurooncol. 2014;119(1): 121-128.

28. Cunha MV, Al-Omair A, Atenafu EG, et al. Vertebral compression fracture (VCF) after spine stereotactic body radiation therapy (SBRT): analysis of predictive factors. Int $J$ Radiat Oncol Biol Phys. 2012;84(3):e343-e349.

29. Dunne EM, Fraser IM, Liu M. Stereotactic body radiation therapy for lung, spine and oligometastatic disease: current evidence and future directions. Ann Transl Med.2018;6(14): 283.

30. Kothari G, Loblaw A, Tree AC, et al. Stereotactic body radiotherapy for primary prostate cancer. Technol Cancer Res Treat. 2018;17:1533033818789633.

31. Rose PS, Laufer I, Boland PJ, et al. Risk of fracture after single fraction image-guided intensity-modulated radiation therapy to spinal metastases. J Clin Oncol. 2009;27(30): 5075-5079.

32. Myrehaug S, Sahgal A, Hayashi M, et al. Reirradiation spine stereotactic body radiation therapy for spinal metastases: systematic review. J Neurosurg Spine. 2017;27(4):428-435.

\section{Disclosures}

Dr. Mummaneni: consultant for DePuy Synthes, Globus, and Stryker; direct stock ownership in Spinicity/ISD; royalties from DePuy Synthes, Thieme Publishers, and Springer Publishers; honoraria from Spineart; and non-study-related clinical or research effort from AO Spine, NREF, and ISSG. Dr. Chou: consultant for Medtronic and Globus and royalties from Globus.

\section{Author Contributions}

Conception and design: all authors. Acquisition of data: all authors. Analysis and interpretation of data: all authors. Drafting the article: all authors. Critically revising the article: all authors. Reviewed submitted version of manuscript: Susko, Mummaneni, Braunstein, Chou. Statistical analysis: Vargas.

\section{Supplemental Information \\ Previous Presentations}

Portions of this work were presented as a podium presentation at the 2019 CNS Annual Meeting, San Francisco, California, October 22, 2019.

\section{Correspondence}

Enrique Vargas: School of Medicine, University of California, San Francisco, CA. enrique.vargas@ucsf.edu. 